\title{
Impact of system-level changes and training on alcohol screening and brief intervention in a family medicine residency clinic: a pilot study
}

\author{
James Aaron Johnson ${ }^{1 *}$, James Paul Seale ${ }^{1}$, Sylvia Shellenberger ${ }^{1}$, Maribeth Hamrick ${ }^{2}$ and Robert Lott ${ }^{3}$
}

\begin{abstract}
Background: Although screening and brief intervention (SBI) are effective in reducing unhealthy alcohol use, major challenges exist in implementing clinician-delivered SBI in primary care settings. This 2006-2007 pilot study describes the impact of systems changes and booster trainings designed to increase SBI rates in a family medicine residency clinic which annually screened adults with a self-administered AUDIT-C questionnaire and used paper prompts to encourage physician interventions for patients with positive screens.

Methods: Investigators added the Single Alcohol Screening Question (SASQ) to nursing vital signs forms, added a checkbox for documenting brief interventions to the clinicians' outpatient encounter form, and conducted onehour nurse and clinician booster trainings. Impact was measured using chart reviews conducted before implementing systems changes, then six weeks and six months post-implementation.

Results: At all three time points screening rates using AUDIT-C plus SASQ exceeded 90\%, however AUDIT-C screening decreased to $85 \%$ after 6 months ( $p=.025$ ). Identification of unhealthy alcohol users increased from $4 \%$ to $22.9 \%$ at six weeks and $18.8 \%$ at six months $(\mathrm{p}=.002)$ using both screens. Nursing vital signs screening using the SASQ reached $71.4 \%$ six weeks after implementation but decreased to $45.5 \%$ at six months. Changes in clinician brief intervention rates did not achieve statistical significance.

Conclusions: This is the second study reporting sustained primary care alcohol screening rates of more than 90\%. Screening patients with SASQ and/or AUDIT-C identified a higher percentage of patients with unhealthy alcohol use. Dissemination of effective strategies for identifying unhealthy alcohol users should continue, while future research should focus on identifying more effective strategies for increasing intervention rates.
\end{abstract}

Keywords: Alcohol screening, Medical education, Resident training, Brief intervention, Chart review

\section{Background}

There is strong evidence for the efficacy of screening and brief interventions (SBI) in reducing unhealthy alcohol use, particularly in primary care settings [1,2]. A recent study comparing preventive services found SBI to be the third highest in terms of preventable burden of disease and cost-effectiveness, with high potential for reducing both medical and societal costs related to unhealthy alcohol use [3] as well as alcohol-related health risks [4]. As a result of SBI's efficacy, the US Preventive Services Task Force re-

\footnotetext{
* Correspondence: Johnson.Aaron@mccg.org

'Department of Family Medicine, Mercer University School of Medicine and Medical Center of Central Georgia, 3780 Eisenhower Parkway, Suite 3, Macon, GA 31206, Georgia

Full list of author information is available at the end of the article
}

commends screening all primary care patients and providing behavioral counseling interventions (typically including feedback on their drinking, advice to reduce consumption, and if possible, negotiating a lower drinking goal) to reduce unhealthy alcohol use [5].

Because current levels of SBI delivery are among the lowest among comparable preventive services, the Substance Abuse and Mental Health Services Administration has mounted a major initiative supporting residency training in SBI and referral to treatment (SBIRT) for both alcohol and drugs. [6] While several previous training efforts have resulted in modest increases in rates of advising patients to reduce drinking among practicing physicians [7-9] and in residency training programs [10-13], several 


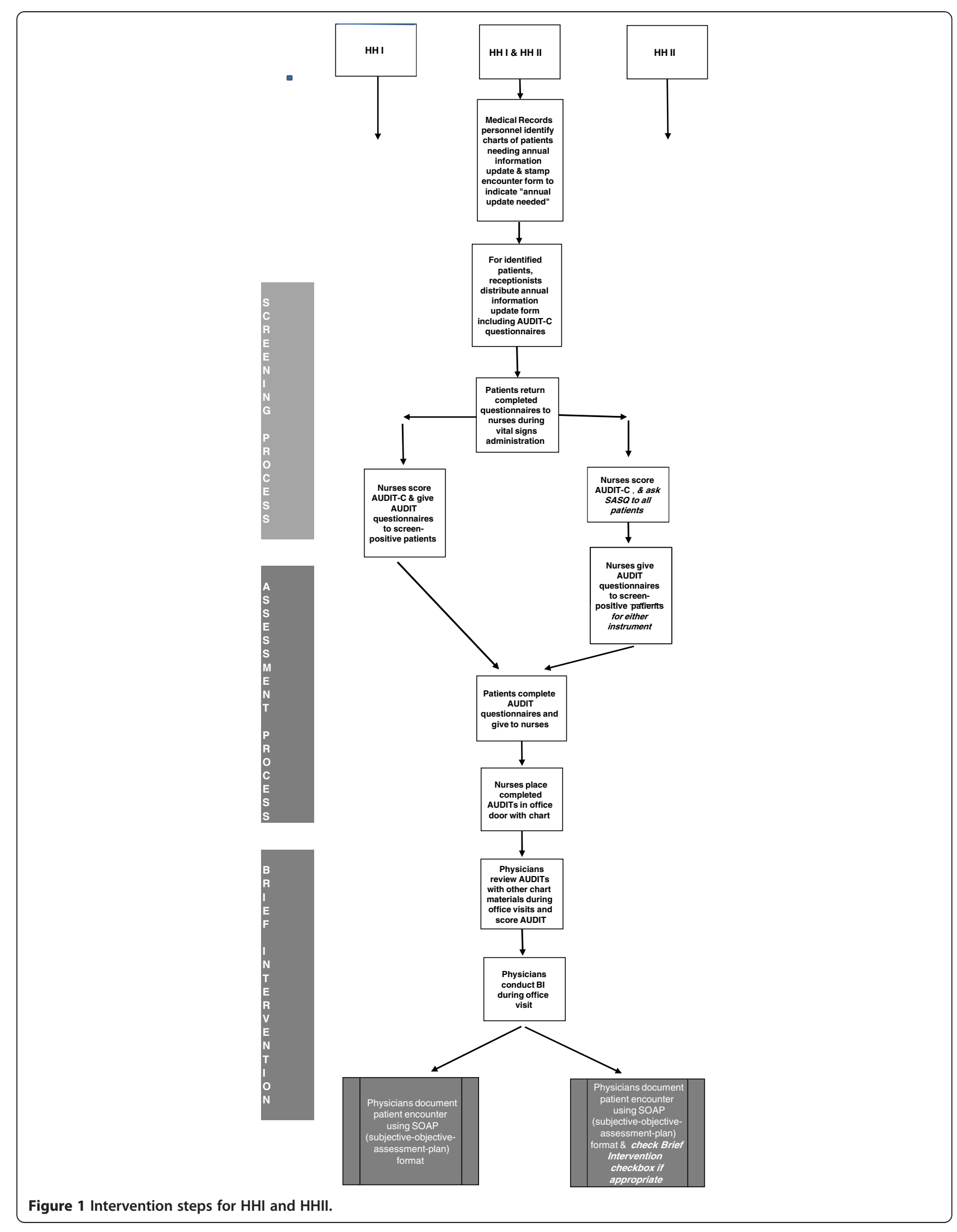


recent studies have found major challenges to increasing primary care clinicians' brief intervention rates despite investment of significant time and resources $[14,15]$.

The Healthy Habits Project (HH1) was a pilot program which resulted in significant increases in alcohol SBI in a family medicine residency training clinic during 20022003 [10]. Details of implementation methods have been previously published [11]. Briefly, clinicians participated in a three-hour training which included a didactic component and skills-based training. The project's approach to SBI service delivery, modeled after the WHO's PHEPA Project and the University of Connecticut's Cutting Back Project $[7,8,16]$, included use of a modified AUDIT-C questionnaire for alcohol screening, inclusion of the AUDIT-C on a self-administered paper health habits questionnaire distributed by clinic receptionists, the scoring of the initial alcohol screen by nurses who also gave screen-positive patients paper AUDIT questionnaires to complete for further assessment, the placement of intervention brochures and other SBI materials at physician workstations throughout the clinic, and providing residents and nurses with performance feedback regarding brief intervention (BI) rates on a monthly basis. Low initial screening rates were addressed by incorporating the AUDIT-C questions into annual self-administered patient information updates required by guidelines of the Joint Commission on Accreditation of Hospital Organizations (JCAHO), distributed by receptionists and completed in the waiting room. After incorporation of AUDIT-C questions into this update form, overall screening rates reached $82 \%$. Alcohol screening was included among other survey questions required by JCAHO because collection of required JCAHO information is a high priority for the clinic's sponsoring hospital, and staff regularly receive feedback designed to ensure that JCAHO requirements are met.

After conclusion of the study, investigators identified several areas for potential project improvement: rates of unhealthy alcohol use (8\%) were well below the $28 \%$ reported by the National Epidemiologic Survey on AlcoholRelated Conditions [17], percentages of unhealthy drinkers receiving brief interventions were less than $50 \%$, and chart reviews revealed limited documentation of SBI services provided [11]. This pilot study examines the impact of quality improvement efforts involving both systems changes and booster trainings to address barriers to SBI service delivery. It was hypothesized that the interventions would increase the rates of patients screened, unhealthy drinkers identified and BIs documented.

\section{Methods}

Healthy Habits II (HH2) was designed and implemented in June 2006 using three specific quality improvement efforts described below:
1) Screening protocol: AUDIT-C annual questionnaire screening as conducted in HH1 continued. In an attempt to increase alcohol screening rates and identification of unhealthy drinkers, clinic procedures were modified to integrate verbal administration of single screening questions for tobacco use and unhealthy drinking into nursing vital signs. For alcohol screening, nursing vital signs templates were modified to include the SASQ, a validated measure for identifying unhealthy drinking advocated by the NIAAA Clinician's Guide $[17,18]$, as well as a simple method for recording responses. Nurses were requested to ask the SASQ at every visit as part of patient vital signs, and to ask all screen-positive patients to self-administer the paper AUDIT questionnaire and give it to their physician. Training sessions emphasized to nurses that the SASQ had been carefully worded and validated and should be asked exactly as written. Sessions included skills practice and discussions of how to manage problems encountered during screening.

2) Modification of clinical encounter charting forms: In an attempt to increase documentation of physician BIs, physicians' clinical encounter forms in the clinic's paper chart system were modified to include checkboxes for documenting brief interventions for alcohol and tobacco. These checkboxes were included in the section of the form where physicians documented their treatment plans. All clinicians were accustomed to routine review of their charts for quality control purposes, but were not specifically informed that this section of the chart would be reviewed.

3) Booster training for nurses and residents: In an attempt to improve rates of brief intervention, residents received a one-hour SBI booster training session timed to coincide with implementation of single question screening. All residents in the residency program had previously received a threehour skills-based seminar, similar to the HH1 training, as part of their orientation at the beginning of their residency training. The $\mathrm{HH} 2$ conference reinforced the importance of BI for unhealthy drinking, taught residents how to interpret responses to the SASQ, reviewed procedures for scoring AUDIT forms and conducting brochure-based BIs, and encouraged residents to use the new checkboxes stating "Advised to stop smoking" and/or "Advised to quit drinking/cut back" when brief interventions were performed. Faculty received an abbreviated orientation to changes in the charting form during faculty meetings. 
Figure 1 provides a side by side comparison of the procedural differences for conducting SBI in $\mathrm{HHI}$ and HHII.

\section{Instruments}

The AUDIT-C is a three-question validated instrument for detecting at-risk and problem drinking [19-21], taken from the ten-question Alcohol Use Disorders Identification Test, developed by the World Health Organization (WHO) [22]. Questions 1 and 2 assess the quantity and frequency of alcohol consumption. Question 3 assesses the frequency of high-volume drinking days. For this study, Question 3 was modified to ask "How often do you have 4 or more drinks on one occasion?" rather than "How often do you have 6 or more drinks on one occasion?" This modification, which was also used in the "Cutting Back" study $[7,8]$, was made based on recommendations in WHO guidelines for use of the AUDIT [23], due to differences in the larger size of the U.S. standard drink (14 g) as compared to the $10 \mathrm{~g}$ standard drink used in the original AUDIT validation. Using a cutoff score of 4 or higher, U.S. studies have found the AUDIT-C to have sensitivity ranging from $76 \%$ to $86 \%$ and specificity ranging from $72 \%$ to $80 \%$ in detecting unhealthy alcohol use [19,24].

The NIAAA SASQ is a single question validated instrument for detecting at-risk and problem drinking advocated for universal screening in primary care by the U.S. National Institute for Alcohol Abuse and Alcoholism ["How many times in the past year have you had. .5 or more drinks in a day (for men) OR 4 or more drinks in a day (for women)"] [17]. Using a cutoff point of one or more times in the past year as a positive screen, SASQ has a sensitivity of $82 \%$ and specificity of $79 \%$ in detecting unhealthy alcohol use in primary care patients. [18]

\section{Measurement methods}

This study was approved by the Mercer University and Medical Center of Central Georgia Institutional Review Boards. Due to limited resources and the pilot nature of this study, a series of three reviews of approximately 100 patient charts was conducted on consecutive adult patients seen by residents in the residency clinic at specific time points. A pre-intervention chart review $(n=95)$ was conducted, beginning one month prior to implementation of $\mathrm{HH} 2$. Post-intervention chart reviews were conducted beginning six weeks $(n=93)$ and six months $(n=100)$ after implementation. These intervals were chosen based on previous observation that quality improvement interventions often produce an immediate change in staff behavior that dissipates over time. Chart reviews assessed whether the 1) AUDIT-C and/or single alcohol screening question were completed, 2) the patient's score on these instruments was positive or negative, and 3) there was evidence in either the clinician's progress notes or check- boxes that a brief intervention was conducted by the physician. BI was defined as any comment indicating alcohol education was given, patients were given advice to reduce or stop drinking, or evidence of referral for detoxification or treatment. Previous studies indicate that clinicians frequently neglect to document both alcohol diagnoses and brief interventions $[10,25]$.

\section{Analyses}

This study design used chi-square tests to identify significant changes across the study's three time points in the percentage of patients being screened by the AUDIT-C and/or SASQ, the percentage screening positive for unhealthy alcohol use, and for those screening positive, the percentage receiving a physician-delivered brief intervention.

\section{Results}

In each of the three chart review samples, the average patient was over 40 (41.4 years, 48.1 years, and 48.4 years, respectively), a majority of patients were African American and females comprised approximately two thirds of those sampled (72.6\%, 59.1\%, 58.0\%, respectively). Gender and ethnicity composition of study patients was similar to published data regarding the clinic's overall demographic pattern [26]. A comparison of demographic characteristics across the three time points (see Table 1) shows a significantly lower average age among charts sampled before implementation than 6 weeks and 6 months postimplementation. Gender and race/ethnicity were not significantly different across the three time points.

Table 2 compares rates of screening, detection of unhealthy alcohol use, and brief interventions for the three time points. Available data from the original Healthy Habits 1 study are included for comparison purposes. During the implementation period, the annual screening of patients using the AUDIT-C forms distributed by receptionists continued. The percentage of patients receiving the AUDIT-C at any of the data collection periods remained high, though a chi-square test indicates a statistically significant $10 \%$ decline between 6 weeks and 6 months post system change $(\mathrm{p}=.025)$. SASQ screening rates at six weeks post implementation were $71.4 \%$, however this rate decreased to $45.4 \%$ after six months ( $\mathrm{p}<.001)$. More than $90 \%$ of patients were being screened for unhealthy alcohol use using AUDIT-C and/or SASQ at all three time points, and at 6 months, the percentage of patients being screened by at least one of these instruments was significantly higher when compared to AUDIT-C alone $(90.6 \%$ vs. $85.6 \%$, $\mathrm{df}=95, \mathrm{p}=.025)$.

Among patients screened $(n=267)$, screen positive rates were comparable between AUDIT-C and SASQ at both post-implementation time points. The total percentage of patients identified as screen positive by SASQ and/or 
Table 1 Participating patient demographics across each enrollment period

\begin{tabular}{|c|c|c|c|c|}
\hline & Baseline $(n=95)$ & 6-weeks ( $n=93$ ) & 6- months $(n=100)$ & Chi-square value ( $p$-value) \\
\hline$\%$ Female & $72.6 \%$ & $59.1 \%$ & $58.0 \%$ & $5.455(.065)$ \\
\hline Race/Ethnicity & & & & $5.863(.210)$ \\
\hline - African American & $53.6 \%$ & $50.0 \%$ & $64.0 \%$ & \\
\hline - Caucasian & $44.3 \%$ & $48.9 \%$ & $33.0 \%$ & \\
\hline - Other & $2.1 \%$ & $1.1 \%$ & $3.0 \%$ & \\
\hline Mean Age in Years $(S D)^{a}$ & $41.4(15.8)$ & $48.1(13.7)$ & $48.4(13.4)$ & $7.268(.001)$ \\
\hline
\end{tabular}

a - One-way ANOVA performed for differences in means yields F-statistic $=7.268$ and $p=.001$.

AUDIT-C was significantly higher than the percentage identified by the AUDIT-C alone at 6 weeks (22.9\% vs. $14.3 \%, \mathrm{df}=82, \mathrm{p}=.004)$. At 6 months, the difference in screen positive rates was not statistically significant $(18.8 \%$ vs. $14.1 \%$, df- $68, \mathrm{p}=.083)$. Using the combination of AUDIT-C and/or SASQ to screen patients resulted in a significant increase in the identification of unhealthy drinking in the post-implementation periods to $22.9 \%$ and $18.8 \%$, respectively $(\mathrm{p}=.002)$, when compared to the baseline screen-positive rate (4.4\%). Though the data in Table 2 appear to show an increase in brief intervention rates from baseline (25\%) to 6 weeks $(52.4 \%)$ and 6 months (61.1\%), the change between baseline and post implementation measures is not statistically significant $(\mathrm{df}=68$, $\mathrm{p}=.083$ ), perhaps due to limited sample size and the small number of positive screens at pre-implementation $(n=4)$.

\section{Discussion}

\section{Screening rates}

$\mathrm{HH} 2$ interventions did not significantly increase the percentage of patients receiving alcohol screening, perhaps because baseline screening rates were already high, leaving little room for increase. While universal screening (i.e. $100 \%)$ is desirable, reaching $90+$ percent of patients with one or more standardized screening instruments is an acceptable outcome and one that has not been replicated in many studies of SBI implementation.

\section{Detection of unhealthy alcohol use}

Combined use of AUDIT-C and/or SASQ detected higher numbers of unhealthy drinkers than either instrument alone. This finding is consistent with two recent validation studies which found higher sensitivity for detecting unhealthy alcohol use by combining use of a single question to screen for heavy drinking with AUDIT screening $[27,28]$. The percentage of unhealthy drinkers identified by the SASQ varied over time. During the project's initial 6 weeks, the SASQ achieved the highest screen-positive rate measured by any single instruments alone--22.9\%, a rate which is only slightly lower than the $28 \%$ rate found in NESARC survey [17]. For reasons that are unclear these rates were not sustained. Possible explanations include nurses changing the way questions were asked in light of patient dissatisfaction or patients changing their answers to avoid having to undergo further assessment and BI. Another curious finding from this study is the low screen-positive rate on the AUDITC (4.4\%) during the baseline period. Demographic differences between the baseline chart review and subsequent chart reviews, including the lower mean age of patients and higher percentage of female participants, could account in

Table 2 Screening, unhealthy drinking, and intervention rates across each enrollment period

\begin{tabular}{|c|c|c|c|c|c|c|}
\hline & & HH1 (2003) & $\begin{array}{l}\text { Baseline } \\
(\mathrm{n}=94)\end{array}$ & $\begin{array}{l}\text { 6-weeks } \\
(\mathrm{n}=86)\end{array}$ & $\begin{array}{l}\text { 6- months } \\
(\mathrm{n}=97)\end{array}$ & $\begin{array}{l}\text { Chi-square } \\
\text { Value }^{\mathrm{a}} \text { ( } p \text {-value) }\end{array}$ \\
\hline \multirow[t]{3}{*}{ Screening Rate } & AUDIT-C & $n / a$ & $94.7 \%$ & $95.3 \%$ & $85.6 \%$ & $7.393(p=.025)$ \\
\hline & SASQ & - & - & $71.4 \%$ & $45.4 \%$ & $13.123(p<.001)$ \\
\hline & $\begin{array}{l}\text { AUDIT-C and/or } \\
\text { SASQ }\end{array}$ & $\mathrm{n} / \mathrm{a}$ & $94.7 \%$ & $97.6 \%$ & $90.6 \%$ & $3.991(p=.136)$ \\
\hline Patients screened & & $n=3014$ & $(n=91)$ & $(n=84)$ & $(n=92)$ & \\
\hline Positive Rate & AUDIT-C & $8.0 \%$ & $4.4 \%$ & $14.3 \%$ & $14.1 \%$ & $5.962(p=.051)$ \\
\hline \multirow[t]{2}{*}{ (\% of Screened) } & SASQ & -二-二- & - & $22.4 \%$ & $13.6 \%$ & $1.326(p=.250)$ \\
\hline & $\begin{array}{l}\text { AUDIT-C and/or } \\
\text { SASQ }\end{array}$ & $8.0 \%$ & $4.4 \%$ & $22.9 \%$ & $18.8 \%$ & $13.004(p=.002)$ \\
\hline Screen positives & & $\mathrm{n}=241$ & $(n=4)$ & $(n=21)$ & $(n=18)$ & \\
\hline $\begin{array}{l}\text { Intervention Rate (\% of Screen } \\
\text { Positives) }\end{array}$ & & $47.7 \%$ & $25.0 \%$ & $52.4 \%$ & $61.1 \%$ & $1.736(p=.420)$ \\
\hline
\end{tabular}

${ }^{\mathrm{a}}$ Chi-square test statistic with $2^{\circ}$ of freedom. 
part for this difference. The analyses presented here did not allow for control of demographics as potentially confounding variables. Previous research, conducted in primary care settings, across diverse groups of patients has found single alcohol screening questions and the AUDIT-C to have similar sensitivity when both were administered verbally in confidential studies[18,21,24,27,29]. Bradley et al. previously compared verbal administration of the AUDIT-C in the clinical setting with self-administered AUDIT-C questionnaires collected through a mail survey, and found clinical screening to be less effective in identifying unhealthy drinkers [30]. This study is one of the first to compare verbal administration of a single question screen with written administration of the AUDIT-C, and found similar levels of detection of unhealthy drinking. While reasons for this finding are unclear, it is possible that it is easier to perform single question screening with high levels of fidelity in the clinical setting, or that patients are less defensive in honestly answering the single question screen. Incorporating both AUDIT-C and SASQ instruments into the clinic increased the detection of unhealthy drinkers by $4.7-8.6 \%$ when compared with AUDIT-C alone and by $0.5-5.2 \%$ when compared to SASQ alone. Larger longitudinal studies are needed to determine whether these differences are sustained over time and whether the increased number of patients detected by using two instruments with overlapping domains and similar sensitivity and specificity warrants the additional cost incurred. Simply adding the SASQ to the AUDIT-C in written form, which would eliminate the use of additional time during nursing vital signs, is a potential screening option to be tested in future studies.

\section{Impact on $\mathrm{BI}$ rates}

During this pilot project, BI rates were more than twice those at baseline, returning to levels slightly above those reported in HH1. While these increases in BI rates did not achieve statistical significance, a retrospective power analysis indicates that the study was underpowered to detect this level of difference in BI rates. With particularly low rates of screen positives at baseline, the sample of charts would need to be more than three times as large to detect a significant change in BI rates. With limited resources, a study of that size was not feasible.

This study utilized booster trainings and performance feedback to improve BI rates, in contrast to $\mathrm{HH} 1$, which had included an initial training plus performance feedback but no booster trainings. As the search continues for effective strategies for achieving and maintaining high BI rates, a potential area for future study is comparing the impact of booster trainings on BI rates versus performance feedback versus a combination of these two approaches.

\section{Systems issues}

In an attempt to create a sustainable system that would consistently identify a high percentage of unhealthy drinkers, $\mathrm{HH} 1$ and $\mathrm{HH} 2$ tested attempts to link alcohol screening to two different "universal" proceduresannual self-administered information updates, which are required by JCAHO, and nursing vital signs, which are routinely performed on all patients. In this setting, use of annual self-administered screening questionnaires was the single approach which achieved the highest screening rate, with high levels of acceptability among both patients and clinic staff. Single question screening during nursing vital signs at every clinic visit proved to be problematic. While asking nurses to screen patients at every visit avoided the challenge of creating a reminder system that would prompt nurses at a specific interval (for example, once a year), both nurses and patients described dissatisfaction with verbal screening at every visit. While initial screening rates were high, over six months there were declines in both completion rates by nurses $(71 \%$ to $45 \%)$ and in screen-positive rates $(22 \%$ to $14 \%)$.

Reasons for the decline in self-administered questionnaire screening rates (from 93\% to 85\%) at 6 months are unclear, but could be related to the increased attention being given to verbal administration of the SASQ. Nonetheless, screen-positive rates were relatively high $(14 \%)$ at both post-intervention measurement points and screening rates exceeded $85 \%$ at every time point, demonstrating both the effectiveness of linking alcohol screening to information required by JCAHO and high acceptance of this self-administered screening approach to both patients and clinic staff. This is the second study to achieve ongoing alcohol screening rates of $85 \%$ or higher. The Veterans Administration outpatient system achieved an annual AUDIT-C alcohol screening rate of $93 \%$ after adoption of a mandatory performance measure for alcohol screening in 2003 that also included training of quality managers, use of a computerized clinical reminder with automatic scoring of the AUDIT-C questionnaire, monitoring by both medical record reviews and patient satisfaction questionnaire, and financial incentives for high performance [31]. This study, conducted in a clinic without an electronic medical record, achieved these results by combining nurse and clinician training with a different kind of systems change-tying alcohol screening to a carefully-monitored systems process which was linked to an accreditation process that generates high levels of monitoring and compliance by many U.S. healthcare facilities. It is noteworthy that, in contrast to declines in BI rates, alcohol screening rates, which reached $82 \%$ by the end of $\mathrm{HH} 1$ [11], actually increased during the three years following the HH1 study, despite 
the absence of booster trainings and performance feedback.

\section{Limitations}

Some differences observed in this study could be due to differences in patient demographics, rather than the project's systems interventions. As a small pilot study in a single clinic, the results of the study may not be generalizeable to other primary care clinics. Larger multi-site studies are needed to determine if results are replicable. Likewise, clinic staff members were aware that their screening and brief intervention activity was being observed and documented. This could have resulted in a Hawthorne effect whereby the positive changes in screening rates were largely the result of study itself.

\section{Conclusions}

This study indicates that alcohol screening using the SASQ and/or AUDIT-C can increase identification of unhealthy drinkers. Results of this study and others [30-32] suggest that effective strategies have now been developed that can achieve alcohol screening rates of $90 \%$ or higher, and that efforts to disseminate such strategies more widely are needed. Greater attention now needs to be directed to methods for increasing BI rates, which may require different strategies. BI rates in $\mathrm{HH} 1$ and $\mathrm{HH} 2$ compare favorably with most SBI efforts analyzed in a recent review by Williams et al. [32]. The drop in BI rates between HH1 and $\mathrm{HH} 2$, followed by SBI increases during this pilot project, suggest the value of interventions such as performance feedback and booster trainings in the face of the constant competing demands of primary care [33]. Future prospective studies of these and other systems changes, as well as national initiatives such as JCAHO-sponsored performance measures and financial practice incentives, may help elucidate the most effective means of delivering this valuable preventive service in primary care.

\section{Competing interests}

No financial disclosures or other conflicts of interest were reported by the authors of this paper.

\section{Authors' contributions}

JAJ conducted the data analysis and led the writing of the manuscript. JPS designed the study and assisted with the writing of the manuscript. SS assisted with the study design and contributed to the development of the manuscript. MBH and RL both conducted data collection and provided feedback on the content of the manuscript. All authors read and approved the final manuscript.

\section{Acknowledgments}

The authors gratefully acknowledge support of the Medcen Foundation in Macon, GA (Seale, Medical Director; Johnson, Project Director; Shellenberger, Director of Evaluations) and Grant \#T1020278 from the Substance Abuse and Mental Health Services Administration (SAMHSA). Neither the Medcen Foundation nor SAMHSA had any role in the development of this study or in the preparation/submission of the manuscript. There was no scientific writer for this manuscript.

\section{Author details}

'Department of Family Medicine, Mercer University School of Medicine and Medical Center of Central Georgia, 3780 Eisenhower Parkway, Suite 3, Macon, GA 31206, Georgia. ${ }^{2}$ Department of Medicine 1161, Vanderbilt University Medical Center, 21st Avenue S, Nashville, TN 37232, USA. ${ }^{3}$ Department of Dermatology, New York Medical College, 1901 1st Ave, New York, NY 10029, USA.

Received: 29 May 2012 Accepted: 8 February 2013

Published: 28 February 2013

\section{References}

1. Bertholet N, Daeppen JB, Wietlisbach V, Fleming M, Burnand B: Reduction of alcohol consumption by brief alcohol intervention in primary care: systematic review and meta-analysis. Arch Intern Med 2005, 165:986-995.

2. Kaner EF, Beyer F, Dickinson HO, Pienaar E, Campbell F, Schlesinger C, Heather N, Saunders J, Burnand B: Effectiveness of brief alcohol interventions in primary care populations. Cochrane Database Syst Rev 2007, 18:CD004148.

3. Solberg LI, Maciosek MV, Edwards NM: Primary care intervention to reduce alcohol misuse ranking its health impact and cost effectiveness. Am J Prev Med 2008, 34:143-152

4. Rose HL, Miller PM, Nemeth LS, Jenkins RG, Nietert PJ, Wessell AM, Ornstein S: Alcohol screening and brief counseling in a primary care hypertensive population: a quality improvement intervention. Addiction 2008, 103:1271-1280.

5. Whitlock EP, Polen MR, Green CA, Orleans T, Klein J, U.S. Preventive Services Task Force: Behavioral counseling interventions in primary care to reduce risky/harmful alcohol use by adults: a summary of the evidence for the U.S. Preventive services task force. Ann Intern Med 2004, 140:557-568.

6. SBIRT Medical Residency Cooperative Agreements. www.samhsa.gov/ prevention/SBIRT/grantees/medres.aspx.

7. Babor TE, Higgins-Biddle J, Dauser D, Higgins P, Burleson JA: Alcohol screening and brief intervention in primary care settings: implementation models and predictors. J Stud Alcohol 2005, 66:361-368.

8. Babor TF, Higgins-Biddle JC, Higgins PS, Gassman RA, Gould BE: Training medical providers to conduct alcohol screening and brief interventions. Subst Abus 2004, 25:17-26.

9. Anderson P, Laurant $M$, Kaner E, Wensing M, Grol R: Engaging general practitioners in the management of hazardous and harmful alcohol consumption: results of a meta-analysis. J Stud Alcohol 2004, 65:191-199.

10. Seale JP, Shellenberger S, Boltri JM, Okosun IS, Barton B: Effects of screening and brief intervention training on resident and faculty alcohol intervention behaviours: a pre- post-intervention assessment. BMC Fam Pract 2005, 6:46.

11. Seale JP, Shellenberger S, Tillery WK, Boltri J, Vogel R, Barton B, McCauley M: Implementing alcohol screening and intervention in a family medicine residency clinic. Subst Abus 2005, 26:23-31.

12. Saitz R, Horton NJ, Sullivan LM, Moskowitz MA, Samet JH: Addressing alcohol problems in primary care: a cluster randomized, controlled trial of a systems intervention. The screening and intervention in primary care (SIP) study. Ann Intern Med 2003, 138:372-382.

13. Adams A, Ockene JK, Wheller EV, Hurley TG: Alcohol counseling: physicians will do it. J Gen Intern Med 1998, 13:692-698.

14. Chossis I, Lane C, Gache P, Michaud PA, Pécoud A, Rollnick S, Daeppen JB: Effect of training on primary care residents' performance in brief alcohol intervention: a randomized controlled trial. J Gen Intern Med 2007, 22:1144-1149.

15. Rose GL, Plante DA, Thomas CS, Denton $\sqcup$, Helzer JE: Utility of prompting physicians for brief alcohol consumption intervention. Subst Use Misuse 2010, 45:936-950.

16. Primary Health Care European Project on Alcohol (PHEPA); www.phepa.net/.

17. National Institute on Alcohol Abuse and Alcoholism (NIAAA): Helping Patients Who Drink Too Much: A Clinician's Guide. Bethesda, MD: National Institutes of Health; 2005.

18. Smith PC, Schmidt SM, Allensworth-Davies D, Saitz R: Primary care validation of a single-question alcohol screening test. J Gen Intern Med 2009, 24:783-788.

19. Bush K, Kivlahan DR, McDonell MB, Fihn SD, Bradley KA: The AUDIT alcohol consumption questions (AUDIT-C): an effective brief screening test for problem drinking. Arch Intern Med 1998, 158:1789-1795. 
20. Bradley KA, Bush KR, Epler AJ, Dobie DJ, Davis TM, Sporleder JL, Maynard C, Burman ML, Kivlahan DR: Two brief alcohol-screening tests from the alcohol use disorders identification test (AUDIT): validation in a female veterans affairs patient population. Arch Intern Med 2003, 163:821-829.

21. Bradley KA, DeBenedetti AF, Volk RJ, Williams EC, Frank D, Kivlahan DR: AUDIT-C as a brief screen for alcohol misuse in primary care. Alc Clin Exp Res 2007, 31:1208-1217.

22. Saunders JB, Aasland OG, Babor TF, De la Fuente JR, Grant M: Development of the Alcohol Use Disorders Identification Test (AUDIT): WHO collaborative project on early detection of persons with harmful alcohol consumption-Il. Addiction 1993, 88:791-804.

23. Babor TF, Higgins-Biddle JC, Saunders JB, Monteiro MG: The Alcohol Use Disorders Identification Test: Guidelines for use in primary care. 2nd edition. Geneva: World Health Organization; 2001.

24. Seale JP, Boltri JM, Shellenberger S, Velasquez MM, Cornelius M, Guyinn M, Okosun I, Sumner H: Primary care validation of a single screening question for drinkers. J Stud Alcohol 2006, 67:778-784.

25. Mitchell AJ, Meader N, Bird V, Rizzo M: Clinical recognition and recording of alcohol disorders by clinicians in primary and secondary care: metaanalysis. Brit J Psychiatry. 2012, 201:93-100.

26. Seale JP, Davis-Smith M, Okosun I: Ethnic and gender differences in lifestyle risk factors in a bi-ethnic primary care sample: prevalence and clinical implications. Ethn Dis 2006, 16:460-467.

27. Johnson JA, Lee A, Vinson D, Seale JP: Use of AUDIT-based measures to identify unhealthy alcohol use and alcohol dependence in primary care: a validation study. Alc Clin Exp Res 2012,: doi:10.1111/j.1530-0277.2012.01898.x.

28. McGinnis KA, Justice AC, Kraemer KL, Saitz R, Bryant KJ, Fiellin DA: Comparing alcohol screening measures among HIV-infected and uninfected men. Alc Clin Exp Res 2012: doi:10.1111/j.1530-0277.2012.01937.x.

29. Frank D, DeBenedetti AF, Volk RJ, Williams EC, Kivlahan DR, Bradley KA: Effectiveness of the AUDIT-C as a screening test for alcohol misuse in three race/ethnic groups. J Gen Intern Med 2008, 23:781-787.

30. Bradley KA, Lapham GT, Hawkins EJ, Achtmeyer CE, Williams EC, Thomas RM, Kivlahan DR: Quality concerns with routine alcohol screening in VA clinical settings. J Gen Intern Med 2011, 26:299-306.

31. Bradley KA, Williams EC, Achtmeyer EC, Volpp B, Collins BJ, Kivlahan DR: Implementation of evidence-based screening in the Veterans Health Administration. Am J Manag Care 2006, 12:597-606.

32. Williams EC, Johnson ML, Lapham GT, Caldeiro RM, Chew L, Fletcher GS, McCormick KA, Weppner WG, Bradley KA: Strategies to implement alcohol screening and brief intervention in primary care settings: A structured literature review. Psychol Addict Behav 2011, 25:206-214.

33. Lapham GT, Achtmeyer CE, Williams EC, Hawkins EJ, Kivlahan DR, Bradley KA: Increased documented brief alcohol interventions with a performance measure and electronic decision support. Med Care 2012, 50:179-187.

doi:10.1186/1747-597X-8-9

Cite this article as: Johnson et al:: Impact of system-level changes and training on alcohol screening and brief intervention in a family medicine residency clinic: a pilot study. Substance Abuse Treatment, Prevention, and Policy 2013 8:9.

\section{Submit your next manuscript to BioMed Central and take full advantage of:}

- Convenient online submission

- Thorough peer review

- No space constraints or color figure charges

- Immediate publication on acceptance

- Inclusion in PubMed, CAS, Scopus and Google Scholar

- Research which is freely available for redistribution

Submit your manuscript at www.biomedcentral.com/submit 